\title{
Aligned Porous Beads Prepared by Frozen Polymerization of Emulsion-Templates Involving Tiny Emulsifier
}

\author{
Zhen $\mathrm{LU}^{1, \mathrm{a}}$, Yun ZHU ${ }^{1, \mathrm{~b}}$, Shengmiao ZHANG ${ }^{1,{ }^{*} \mathrm{c}}$ and Jianding $\mathrm{CHEN}^{1, \mathrm{~d}}$ \\ ${ }^{1}$ School of Materials Science and Engineering, East China University of Science and Technology, \\ Shanghai 200237, China; \\ a719911702@qq.com ${ }^{1}$, ${ }^{\mathrm{a}}$ zhuyun@ecust.edu.cn, ${ }^{\mathrm{c}}$ shmzhang@ecust.edu.cn, \\ jiandingchen@ecust.edu.cn
}

\begin{abstract}
High internal phase emulsion (HIPE) templated porous materials are attracting increasing interests due to its high porosity and tunable structure. However, large amounts $(5-50 \mathrm{vol} \%)$ of suitable non-ionic surfactants are commonly required to stabilize conventional HIPE due to the high internal volume fraction of HIPE. In this work, applying frozen polymerization in HIPE, aligned porous beads were prepared with tiny surfactant $(\sim 0.1 \mathrm{wt} \%)$. These interconnected aligned porous beads were prepared through directional freezing, and frozen ultraviolet (UV) initiation of an oil-in-water $(\mathrm{o} / \mathrm{w})$ HIPE. The HIPEs are extruded by needle, and then directionally frozen in liquid nitrogen to form beads. The frozen beads were exposed under UV irradiation in a $-20^{\circ} \mathrm{C}$ ethanol bath to initiate the monomers in the aqueous phase. Moreover, the morphology of the resulting porous beads were tailored by vary the ratio of oil/water and the amount of emulsifier.
\end{abstract}

\section{Introduction}

Porous polymers are attracting much interest as a result of their application (both real and potential) in many areas of advanced materials science [1] including drug delivery, tissue engineering, chromatography and water treatment. Porous polymers are a subset of porous materials that take advantage of the ease of processability associated with polymers to generate monoliths, films, and beads, often with well-defined porosities and high specific surface areas (SSAs) [2]. High internal phase emulsions (HIPEs) have been found considerable use in materials science as templates to create highly porous structures [3]. Such materials, formed by curing the continuous, or non-droplet, phase of the emulsion, are known as PolyHIPEs. Conventional HIPEs are commonly stabilized by large amounts (5$50 \%$ relative to continuous phase) of suitable non-ionic surfactants due to the high internal volume fraction [4], which is not environmental friendly and is the most cost factor. Although great efforts have been done to eliminate or reducing the amount of the surfactant in the preparation of HIPEs, such as particle-stabilized and CTAB-stabilized HIPEs [5],

\footnotetext{
*Corresponding author:719911702@qq.com
} 
HIPE stabilized by tiny non-ionic surfactant is still highly desired, due to the easy removal of the residue surfactant from the resulting porous materials. Besides, traditionally polymerization of HIPEs was initiated by heating the emulsion system. However, high temperature would destabilize the HIPE by enhancing the Ostwald ripening and coalescence in the emulsion [6].

Alternatively, UV-initiated cryopolymerization (Frozen polymerization) has been widely used to prepare polymeric materials [7].However, there were few report on UVinnitiated HIPEs [ 8]. UV-initiated can be operated under low temperature and the time of reaction can be greatly shortened as well as simply process. Combined frozen HIPE template and UV-initiated method can be complementary advantages.

In this work, by using ice-templated assembly and UV-initiated frozen polymerization of $\mathrm{O} / \mathrm{W}$ HIPEs, the PolyHIPEs beads having both well-defined porous structure and orientation interconnectivity structure in voids' wall. And the morphology of the beads was tuned by changing oil: water ratio and the amount of emulsifier in the continuous phase.

\section{Experimental Section}

\subsection{Materials}

N-isopropylacrylamide(NIPAAm, Tokyo into industrial Co.,Ltd) was recrystallized twice in cyclohexane, N,N'-Methylenebisacrylamide(MBAM,98\%, Sinopharm Chemical Reagent Co.,Ltd), Polyoxyethylene (60) sorbitan monostearate(Tween 60, Sinopharm Chemical Reagent Co.,Ltd), cyclohexane (Shanghai Lingfeng Chemical reagent Co.,Ltd), Diphenyl ketone(Shanghai Lingfeng Chemical reagent Co.,Ltd), and water was fresh deionized.

\subsection{Preparation and Characterization of HIPE and PolyHIPE}

The monomer NIPAAm and MBAM (10:1, wt/wt) mixed with appropriate amount of $\mathrm{H}_{2} \mathrm{O}$ and a small amount of emulsifier $(0.1 \mathrm{wt} \%-2.0 \mathrm{wt} \%)$ using sonic oscillation as aqueous phase were added to a $50 \mathrm{~mL}$ beaker and stirred with a magnetic stirrer at $600 \mathrm{rpm}$ at $25{ }^{\circ} \mathrm{C}$. Cyclohexane as the oil phase containing photoinitiator Diphenyl ketone $(2 \mathrm{wt} \%$ of monomer) was added to the aqueous phase gradually with $30 \mathrm{~s}$. The total emulsion volume was kept at $10 \mathrm{ml}$. The mixture was further stirred at $900 \mathrm{rpm}$ for $3 \mathrm{~min}$ after the addition of oil phase was completed. The HIPEs were then moved into needle and extruded at slow and constant speed to form the beads. And the beads directly dropped into the liquid nitrogen and compeleted shaped. The frozen beads were then placed under UV light $(\lambda=367 \mathrm{~nm})$ for $6 \mathrm{~h}$ to complete the in situ radical cryopolymerization in a $-20{ }^{\circ} \mathrm{C}$ ethanol bath. The resulting polyHIPEs beads (Fig. 1) were dried in freeze drying machine for 3 days.

The stability of HIPEs was evaluated by measuring the backscattering of monochromatic light $(\lambda=880 \mathrm{~nm})$ from the suspension employing an optical analyzer, turbiscan Lab Expert (Formulaction, France). The backscattering of the light from HIPEs was determined by the internal phase volume ratio and the size of the droplets in HIPEs. If the measured HIPEs possess the same internal phase volume ratio, the higher the backscattering percent is obtained, the smaller droplet size and hence the higher stability the HIPE have [9]. So the stability of the emulsion can be reflected through the change of the backscattering light intensity. The morphology of porous beads obtained was detected by a Hitachi S-3400 SEM. The average void diameters and its distribution of the polyHIPEs were performed using the image analysis software Image J (NIH image). 
TABLE 1 PARAMETERS FOR ALIGNED POROUS BEADS PREPARATION AND THE BASIC RESULTING MATERIAL'S PROPERTIES

\begin{tabular}{ccccccc}
\hline No. & $\mathrm{O}: \mathrm{W}^{\underline{a}}$ & $\begin{array}{c}\text { Monomer } \\
\text { content in } \\
\text { aqueous phase } \\
(\%)\end{array}$ & $\begin{array}{c}\text { Emulsifier content } \\
\text { in aqueous phase } \\
(\%)\end{array}$ & $\begin{array}{c}\text { Average } \\
\text { void } \\
\text { diameter } \\
(\mu \mathrm{m})\end{array}$ & $\begin{array}{c}\text { Void } \\
\text { diameter } \\
\text { distribution }\end{array}$ & $\begin{array}{c}\text { Gap of } \\
\text { orientation } \\
(\mu \mathrm{m})\end{array}$ \\
\hline 1 & $80: 20$ & 20 & 0.1 & 50.11 & 10.69 & 4.06 \\
2 & $75: 25$ & 20 & 0.1 & 48.60 & 14.31 & 1.54 \\
3 & $70: 30$ & 20 & 0.1 & 42.02 & 14.73 & 1.90 \\
4 & $75: 25$ & 20 & 0.5 & 34.09 & 11.57 & 1.45 \\
5 & $75: 25$ & 20 & 2 & 24.97 & 6.49 & - \\
\hline
\end{tabular}

${ }^{a} \mathrm{O}: \mathrm{W}$ means the volume ratio of internal phase and external phase

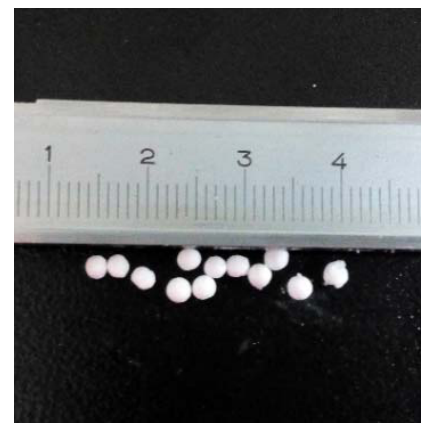

Fig. 1 Photograph of aligned porous PolyHIPEs beads.

\section{Results and Discussion}

As shown in Fig. 2 b, as the time increasing, the HIPE prepared with $0.1 \mathrm{wt} \%$ of Tween 60 underwent sedimentation firstly and then separation. The backscattering intensity of the ends $(0-5 \mathrm{~mm})$ increased firstly and then decreased quickly. And curves reflected the intensity of backscattering became weakened which was consistent with the delamination phenomenon of the HIPE (Fig.2 (a) after one day). All of the results showed that the HIPE prepared with $0.1 \mathrm{wt} \%$ is not suitable to be an emulsion template for preparation of polyHIPE.

In this work, in attempt to address this phenomenon, the HIPE with $0.1 \mathrm{wt} \%$ was frozen via liquid nitrogen as soon as it was prepared. Fig. 4 showed that relatively uniform pore diameter of porous structure were obtained. This is attributed to frozen template possesses the advantages that fixed morphology in short time just through simple physical frozen affect, which prevent the droplets coalescence result in unstable stratification phenomenon. In addition, UV light initiation can be operated under low temperature which ensured the reservation of the morphology. 

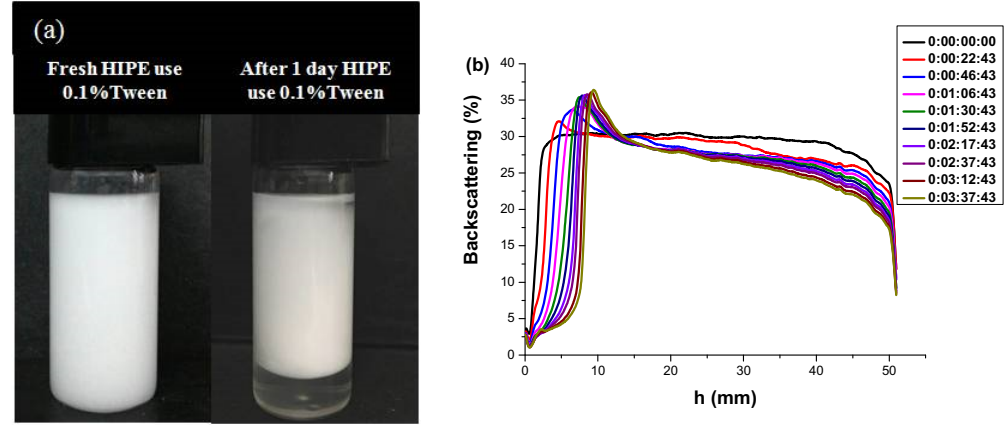

Fig. 2 (a) Photograph of the o/w HIPE with low emulsifier content ( $0.1 \mathrm{wt} \%$ continuous phase). (b) Backscattering data of (a), and the data are represented as a function of time (0:00-03:30 h) and of sample high $(0-50 \mathrm{~mm})$.
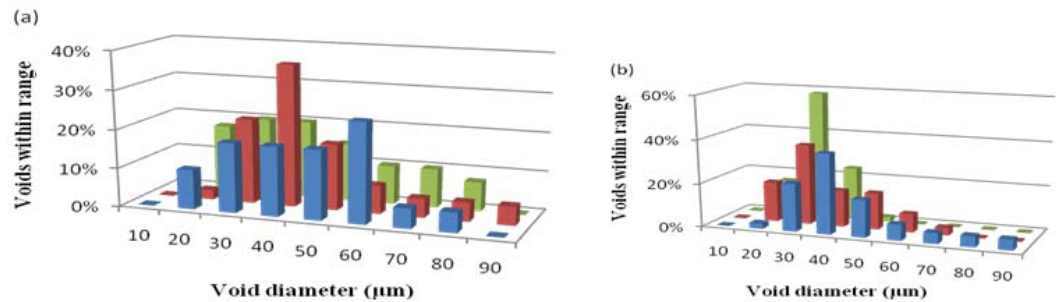

Fig. 3 (a) The effect of the ratio of (o: w) and (b) the content of emulsifier on void diameter distribution, (a) o: $\mathrm{w}$ ratio (from front to back) : 80:20, 75:25 and 70:30; (b) emulsifier content in aqueous phase (from front to back) : $0.1 \mathrm{wt} \%, 0.5 \mathrm{wt} \%$ and $2 \mathrm{wt} \%$.

\subsection{The Effect of the Ratio of Oil Phase and Water Phase (o:w)}

To adjust the morphology of the polyHIPEs, a series of HIPEs with varied o:w ratio were prepare as shown in Table 1. The $\mathrm{o}$ : $\mathrm{w}$ ratio is one of $\mathrm{t}$ major roles in destabilization of HIPEs [10]. Changing he internal phase fraction from 80 to 75 and $70 \mathrm{vol} \%$ was investigated in this work. It was found that decreasing internal phase fraction of the HIPEs decreased the average void diameter of the resulting porous polymer (Fig.3 (a)), however broadened the void diameter distribution (Fig.4 Sample1, 2, 3). When the internal phase was decreased, it could be envisaged that because of the smaller surface over which the emulsifier had to spread and taking into account that the amount of emulsifier (kept constant relative to the continuous phase) increased, the HIPE stability increased [11]. Increasing HIPE stability resulted in a smaller average droplet size.

The oil: water ratio also caused different well-define morphologies. As shown in Fig.4 (Sample1, 2, 3) that increasing continuous phase fraction from 20 to 25 and $30 \%$, the orientation gaps became smaller and interconnectivity reduced obviously. It is attributed to the increasing amount of monomer was polymerized to be the continuous phase. In addition, the orientation structure formation was impeded by the narrow voids which due to that too small void size to reach the critical ice crystal size, and the growth of the ice crystal based on the critical crystal size [12]. 


\subsection{The Effect of Ratio Emulsifier Content in the Continuous Phase}

As shown in Fig.3 (b) and Fig.4 (Sample 2, 4, 5 ) increasing of emulsifier content decreased the void diameter. This is attribute to that the increasing amount of emulsifier spread on the interface between two phases resulted in more stable system which reduces the coalescence of droplets. In addition, it is found that the intermediate emulsifier concentration $(0.5 \mathrm{wt} \%)$ sample presents one of the most obvious orientation morphology. It maybe also contributed by the stable system. However with the increasing to $2 \%$, the voids were too small and impeded the growth of ice crystal.
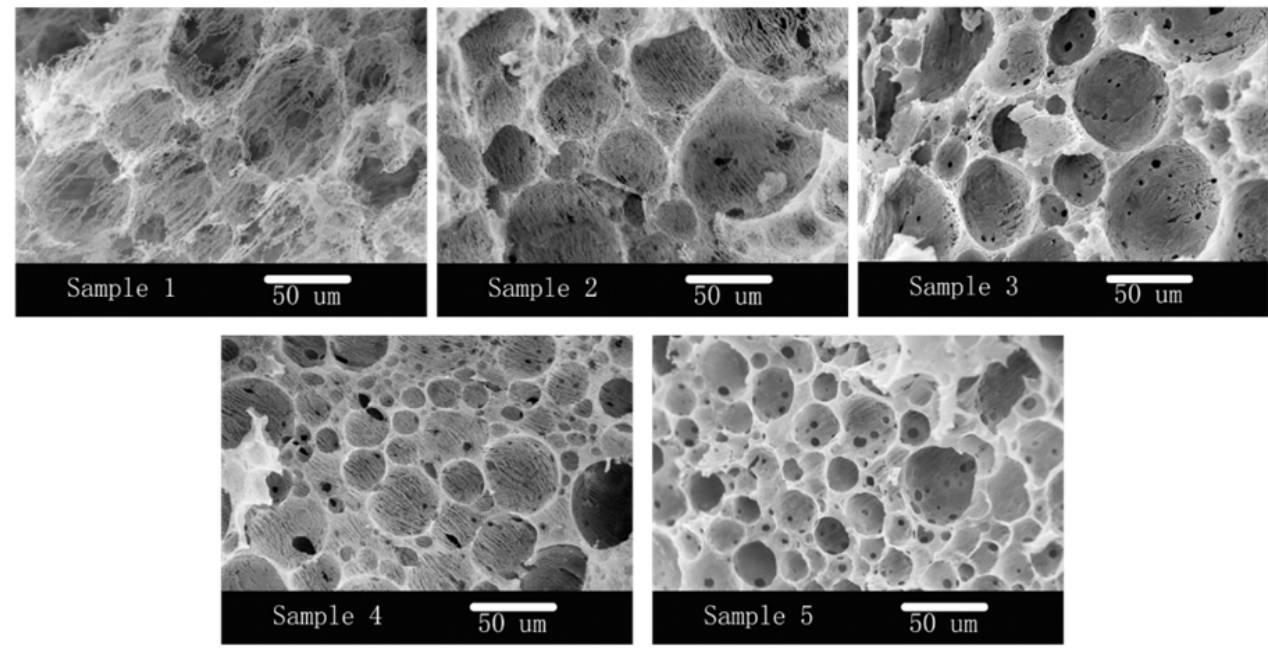

Fig. 4 SEM images for aligned porous materials prepared from O/W HIPE templates.

\section{Summary}

Porous beads, having a well-defined open-cell structure, were prepared based on the HIPE stabilized by low to $0.1 \mathrm{wt} \%$ Tween 60 . The porous beads were prepared by freezing and UV initiating the HIPE templates. In addition, the morphology of these beads could be simply tailored by controlling the ratio of oil: water and the emulsifier concentration in the aqueous phase.

\section{Acknowledgements}

This research was supported by the National Natural Scientific Foundation of China (51203048, 51103042), the Fundamental Research Funds for the Central Universities, Innovation Program of Shanghai Municipal Education Commission (12ZZ056). 


\section{References}

1. M.T. Gokmen, F.E.D. Prez, Porous polymer particles-A comprehensive guide to synthesis, characterization, functionalization and applications, Prog. Polym. Sci. 37 (2012) 365-405.

2. M.S. Silverstein, PolyHIPEs: Recent advances in emulsion-templated porous polymers, Prog. Polym. Sci. 39 (2014) 199-234.

3. N.R. Cameron, Polymerized high internal phase emulsion monoliths, J. Chromatogr. L. 67 (2003) 255-276.

4. N.R. Cameron, High internal phase emulsion templating as a route to well-defined porous polymers, Polymer 46 (2005) 1439-1449.

5. S.M. Zhang, J.D. Chen, V.T. Perchyonok, Stability of high internal phase emulsions with sole cationic surfactant and its tailoring morphology of porous polymers based on the emulsions, Polymer 50 (2009) 1723-1731.

6. M.G. Freire, A.M.A. Dias, M.A.Z. Coelho, J.A.P. Coutinho, I.M. Marrucho, Aging mechanisms of perfluorocarbon emulsion using image analysis, J. Colloid. Interf. Sci. 286 (2005) 224-232.

7. Y.D. Hazan, Porous ceramics, ceramic/polymer, and metal-doped ceramic/polymer nanocomposites via freeze casting of photo-curable colloidal fluids, J. Am. Ceram. Soc. 95 (2012) 177-187.

8. L.Y. Liu, W.T. Yang, Inverse emulsion polymerization of acrylamide intiated by UV radiation, Acta. Polym. Sinica. 4 (2004) 545-553.

9. O. Mengual, et al. TURBISCAN MA 2000: multiple light scattering measurement for concentrated emulsion and suspension instability analysis, Talanta 50 (1999) 445-456.

10. Y. Zhu, S.M. Zhang, Y. Hua, J.D. Chen, C.P. Hu, Hydrophilic porous polymers based on high internal phase emulsions solely stabilized by poly(urethane urea) nanoparticles, Polymer 51 (2010) 3612-3617.

11. B.P. Binks, Particles as surfactants similarities and differences, Curr. Opin. Colloid. In.7 (2002) 21-41.

12. M.F. Song, D.P. Liu, Z.M. Wu, Z.H. Hua, Study on nucleation and growth mechanism of snow crystal, Int. J. Refrig. 25 (2004) 46-50. 